\title{
TEMA -2016: Terapia de presión negativa como técnica de cierre abdominal temporal en el manejo de abdomen abierto
}
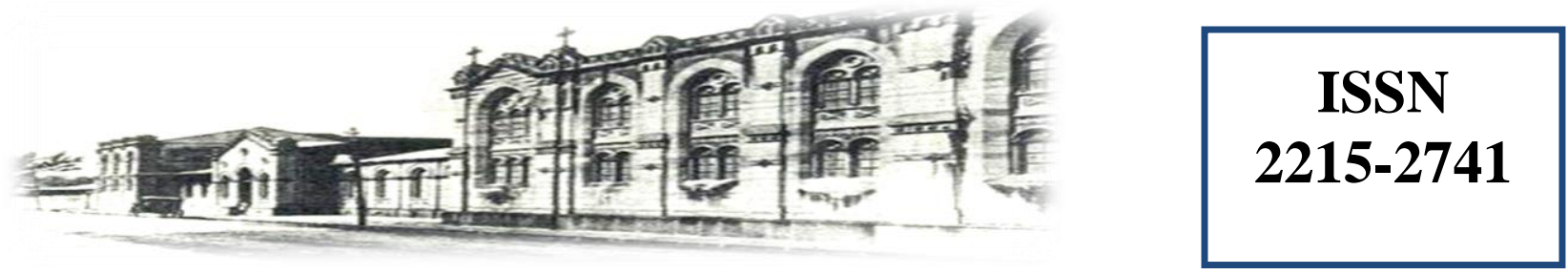

Hospital San Juan de Dios, San José, Costa Rica. Fundado en 1845

Recibido:

$26 / 07 / 2016$

Aceptado:

$06 / 10 / 2016$

\author{
${ }^{1}$ Huixian $\mathrm{Hu}$ Liang \\ ${ }^{2}$ Jairo Sandoval Vargas \\ ${ }^{3}$ José Pablo Castro Álvarez \\ ${ }^{4}$ José Alberto Ayi Wong
}

${ }^{1}$ Médico y Cirujano. Universidad Latina de Costa Rica. Hhu2986@gmail.com

${ }^{2}$ Médico y Cirujano. Universidad Autónoma de Centroamérica. Jairosv1522@gmail.com

${ }^{3}$ Médico Interno. Universidad Autónoma de Centroamérica. jpcastro1989@ hotmail.com

${ }^{4}$ Médico Cirujano. Asistente Especialista en Cirugía General. Hospital San Juan de Dios. jo-

seayiw@gmail.com

\section{RESUMEN}

Múltiples situaciones clínicas desafiantes pueden requerir dejar la cavidad abdominal abierta posterior a una cirugía, resultando en un abdomen abierto (OA, open abdomen) o laparostoma. Las indicaciones para abdomen abierto son generalmente trauma, sepsis abdominal, pancreatitis aguda severa y en situaciones generales en las que se está desarrollando una situación de hipertensión abdominal, con el objetivo de prevenir el desarrollo de síndrome compartimental abdominal. La Sociedad Mundial de Síndrome Compartimental Abdominal (WSACS) define hipertensión abdominal como una presión intraabdominal (PIA) $>12 \mathrm{mmHg}$ y síndrome compartimental abdominal como una PIA $>20 \mathrm{mmHg}$. La WSACS recomienda el uso de la técnica de Kron modificada como el estándar para la medición de la PIA. Este método evalúa la PIA vía presión vesical utilizando una instilación máxima de $25 \mathrm{~mL}$ de solución salina. La necesidad del cierre abdominal temporal (CAT) es una consecuencia lógica de la descompresión abdominal o tratamiento profiláctico de abdomen abierto. Con la adopción aumentada de técnicas de abdomen abierto ha surgido un incremento en la demanda de métodos de CAT. La terapia de presión negativa (TPN) involucra la aplicación de algún grado de succión a una herida abdominal abierta. A pesar de la variedad de métodos de CAT, el consenso del 2013 de la WSACS realiza una recomendación fuerte de utilizar terapia de presión negativa en pacientes críticos con abdomen abierto. 


\section{PALABRAS CLAVE}

Abdomen abierto, TPN, ABThera, WSACS, V.A.C.

\section{ABSTRACT}

Several challenging clinical situations may need leaving the abdominal cavity open after surgery, resulting in an open abdomen (OA) or laparostomy.The indications for Open Abdomen are: trauma, abdominal sepsis, severe acute pancreatitis, and other situations where an increase in intraabdominal pressure will develop intraabdominal hypertension, and abdominal compartment syndrome. The World Society of the Abdominal Compartment Syndrome (WSACS) defines intra-abdominal hypertension as an intra-abdominal pressure (IAP) higher than $12 \mathrm{mmHg}$, and can develop into abdominal compartment syndrome when IAP is higher than $20 \mathrm{mmHg}$. The WSACS advocates the use of the modified Kron technique as the gold standard of IAP measurement. The Kron method assesses the IAP via bladder pressure measurement, using a maximum instillation of $25 \mathrm{ml}$ of sterile saline solution. The need for temporary abdominal closure (TAC) is a logical consequence of abdominal decompression or prophylactic open abdomen treatment. With the increasing adoption of open abdomen techniques, there has been an increase demand for TAC. Negative pressure wound therapy (NPWT) involves applying some level of suction to an open abdominal wound.

\section{KEY WORDS}

Open abdomen, TPN, ABThera ${ }^{\mathrm{tm}}$, WSACS, V.A.C.

\section{INTRODUCCIÓN}

Múltiples situaciones clínicas desafiantes requieren dejar la cavidad abdominal abierta posterior a una cirugía, resultando en un abdomen abierto (OA) o laparostoma. El edema visceral excesivo, puede prevenir el cierre exitoso de la fascia sin tensión después de una laparotomía, llevando a una indicación inescapable de abdomen abierto. Puede ser también necesario dejar el abdomen abierto después de una laparotomía descompresiva por síndrome compartimental abdominal. Además el OA puede ser parte de una cirugía de control de daños y otras estrategias incluyendo una relaparotomia como por ejemplo una cirugía de segunda mirada por isquemia intestinal.

Los beneficios de manejar pacientes con abdomen abierto incluyen prevención de hipertensión intraabdominal (HIA) y de síndrome compartimental abdominal (SCA), identificación temprana de complicaciones intraabdominales y facilitar la revisión de la cavidad. A pesar de estos beneficios, el mantenimiento de un abdomen abierto crea numerosos retos de manejo como el desarrollo de fístula entro-atmosférica e infección. El mantenimiento prolongado de un abdomen abierto puede también llevar a una re-aproximación reducida de la fascia por retracción de la misma.

Con la adopción aumentada de técnicas de abdomen abierto ha surgido un incremento en la demanda de métodos de Cierre Abdominal Temporal (CAT) para proteger el abdomen abierto durante la fase de tratamiento abierto. Las principales técnicas para CAT son: terapia de heridas con presión negativa (NPWT), método Vacuum-Pack, parche de Whitmann, malla absorbible, zipper, bolsa plástica silo, cierre cutáneo y suturas de retención dinámicas.

Estas técnicas varían en su eficacia con respecto a los rangos de cierre de fascia y rangos de morbilidad y mortalidad. Un número de revisiones sistemáticas han concluido que el parche de Whitmann y la terapia de herida con presión negativa tienen los rangos más altos de cierre de fascia y los más bajos de mortalidad. Por ser una técnica de fácil aplicación, y preservación de la fascia, la terapia de presión negativa se está convirtiendo en la opción dominante para CAT en el paciente con abdomen abierto.

Se revisarán los conceptos básicos de la terapia de abdomen abierto y la evidencia más reciente que compare el uso de terapia de presión negativa con otras técnicas disponibles para el cierre abdominal temporal. ${ }^{1,2}$ 


\section{DISCUSIÓN}

\begin{abstract}
Abdomen Abierto
Historia 1940-1990

En unas de las primeras publicaciones conocidas de la literatura inglesa sobre el manejo del abdomen abierto, Ogilvie describe el uso de "un corte de una doble capa de lona o algodón más pequeño que el defecto en los músculos, y la sutura de este en el sitio con suturas catgut interrumpidas" en heridas abdominales de guerra.
\end{abstract}

En una posterior publicación, Ogilvie propone la misma técnica en el tratamiento de heridas abdominales infectadas, dejando el abdomen abierto después de la operación inicial con el objetivo de cerrarlo solo después de 1-4 días. Steinberg describió el manejo de 14 pacientes con peritonitis aguda generalizada, a quienes se les dejo el abdomen abierto con empaques de gasa en la víscera.

Después de 48-72 horas el empaque fue removido y cables previamente colocados a través de la pared abdominal se enlazan. Un segundo estudio descriptivo por Duff et al. describió el manejo de abdomen abierto como un último recurso para el tratamiento de la sepsis intraabdominal difusa cuando todas las otras opciones de manejo fallaran y el abdomen ya no podía cerrarse. Observaron un rango de mortalidad de $39 \%$ y concluyen que es una técnica factible. Anderson, sin embargo encontró un alto rango de mortalidad de $60 \%$ tratando pacientes con sepsis abdominal utilizando la misma técnica que Steinberg. Se reconocieron problemas como evisceración, fistula entero-atmosférica, perdida de líquido y potencial contaminación así como manejo complejo de la herida.

Se introdujeron nuevas técnicas para hacer frente con los problemas asociados con el manejo del abdomen abierto. Estas técnicas incluían el zipper Marlex®, bolsas plásticas (técnica de Bogotá), hojas adhesivas de velcro, mallas absorbibles y la técnica "sándwich". 3

Definición: Las indicaciones para abdomen abierto son generalmente trauma, sepsis abdominal, pancreatitis aguda severa y situaciones en las que se está desarrollando hipertensión abdominal, con el objetivo de prevenir el desarrollo de síndrome compartimental abdominal. El concepto de control de daños tiene dos componentes básicos; controlar el sangrado y la contaminación de la cavidad abdominal, y dejar el abdomen abierto para descomprimir o facilitar el retorno a una relaparotomía planeada.

Para mantener el dominio abdominal se requiere un cierre temporal abdominal (CAT). Los rangos de mortalidad usualmente son mayores de $30 \%$ dependiendo del cohorte del paciente. Esta compleja situación requiere un manejo multidisciplinario por el cirujano y el equipo de UCI en un proceso estadificado especifico.

\section{Fisiopatología del síndrome comparti- mental abdominal}

La Sociedad Mundial de Síndrome Compartimental Abdominal pactó en el 2004 crear un consenso sobre la definición, diagnóstico y tratamiento del síndrome compartimental abdominal (SCA). El abdomen y la pelvis, aunque anatómicamente distintos, representan un único espacio y por lo tanto deben considerarse como uno en la discusión de la presión intraabdominal (PIA) y SCA.

La cavidad abdomino-pélvico es un espacio cerrado, y la elasticidad de las paredes y la característica de sus contenidos determinan la presión intra-abdominal. La presión intraabdominal es bastante uniforme a través de la cavidad y por lo tanto la medición en cualquier sitio, refleja la cavidad completa. La PIA aumenta con la contracción del diafragma (inspiración) y disminuye con la espiración.

La presión de perfusión abdominal (PPA), análogo a la presión de perfusión cerebral se ha propuesto como un predictor más específico de la perfusión visceral y consecuentemente una meta de intervención. Una PPA meta de $\geq 60 \mathrm{mmHg}$ se asocia con una mejor supervivencia en el escenario de hipertensión intraabdominal y SCA.

La presión intraabdominal normal es en realidad menor a $0 \mathrm{mmHg}$. En condiciones como obesidad mórbida, embarazo, enfermedad hepática, ascitis, la PIA puede estar crónicamente elevada a 10-15 $\mathrm{mmHg}$ sin evidencia de fisiología alterada. Durante la enfermedad o después de una cirugía la 
PIA es mayor en aproximadamente 5-7 $\mathrm{mmHg}$, debido a factores como edema tisular o íleo. El punto en el cual la PIA se convierte en hipertensión intrabdominal ha sido un tema de debate; la definición del consenso se establece en $12 \mathrm{mmHg}$, pues este es el punto menor en el cual los efectos patológicos son notables.

Un sistema gradual fue propuesto por el grupo del Hospital General de Denver en 1996, con el interés de guiar las intervenciones. La definición del consenso de la Sociedad Mundial del Síndrome Compartimental Abdominal varía levemente, y es el siguiente:

- Grado I: $12-15 \mathrm{mmHg}$

- Grado II: $16-20 \mathrm{mmHg}$

- Grado III: $21-25 \mathrm{mmHg}$

- Grado IV: $>25 \mathrm{mmHg}$

El síndrome compartimental abdominal se desarrolla como consecuencia de alteraciones en la perfusión relacionadas a hipertensión intraabdominal (HIA). La definición del consenso seleccionó una PIA > 20mmHg, reconociendo que niveles menores de HIA pueden asociarse con disfunción orgánica, (ver tabla 1).

La vía final común de disfunción orgánica es hipoperfusión. El SCA se clasifica como primario si es el resultado de un proceso fisiopatológico en la cavidad abdomino-pélvica. Puede ser el resultado de sangrado, acumulación de líquido ascítico, un tumor de rápido crecimiento u otra masa, edema retroperitoneal, conjunto de lesiones viscerales, etc. El SCA secundario se refiere al desarrollo de SCA en ausencia de un proceso abdomino-pélvico, así también como en los pacientes que necesitan resucitación masiva por hemorragia, sepsis o en la lesión por isquemia/reperfusión que puede llevar a acumulación masiva de ascitis, y edema intestinal y retroperitoneal por acumulo de líquido extracelular/extravascular.

Cada vez más se reconoce que el SCA secundario es parte iatrogénico por resucitación excesiva con líquidos. ${ }^{4}$

\section{Diagnóstico}

\section{Medición de la presión intraabdominal}

La medición de la PIA es un método simple, barato, seguro y preciso en determinar la presencia de hipertensión intraabdominal. Esta medición puede guiar el manejo del paciente. Históricamente la observación física y la medición de la circunferencia abdominal eran utilizadas para determinar la presencia de HIA.

Estos métodos de medición son imprecisos debido al alto riesgo de variabilidad y baja fiabilidad. El rango de métodos para medir la PIA incluyen catéter intragástrico, intra rectal, de vena cava inferior y un sistema de monitoreo de presión a través de un catéter urinario permanente.

La Sociedad Mundial del Síndrome Compartimental Abdominal (WSACS) recomienda el uso de la técnica de Kron modificada como el estándar para la medición de la PIA. El método de Kron evalúa la PIA vía presión vesical utilizando una instilación máxima de $25 \mathrm{~mL}$ de solución salina, se toma de la siguiente manera:

1. Con el transductor en cero y posicionado en línea con la cresta iliaca y línea media axilar.

2. Con el paciente en posición supina

3. Al final de la espiración

4. Con una instilación de volumen no mayor de $25 \mathrm{ml}$ de solución salina

5. 30-60 segundos después de la instilación para permitir la relajación del músculo detrusor de la vejiga.

La fiabilidad de las directrices de medición intermitente establecidas por la WSACS ha sido cuestionada. Más recientemente, la tecnología de monitoreo continuo de la PIA ha sido sugerido como superior a la técnica intermitente.

El método continuo permite el análisis continuo de la PIA vía vejiga y elimina el riesgo de perder elevaciones de la PIA debido a la sincronización, lo que puede ocurrir con técnicas intermitentes. 
La técnica de medición continua de la PIA requiere la inserción de un catéter de tres vías más caro, lo cual puede ser la causa de su uso limitado.

Figura 2. Sistema de llave con tres vías para medición de la PIA

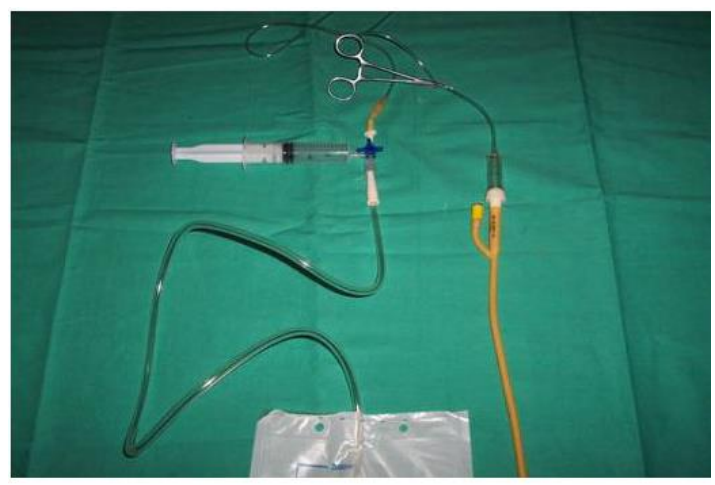

Fuente: Rev Ciencias Médicas v.11 n.1 Pinar del Río ene.-mar. 2007

Hay un pequeño grupo de pacientes en los que la medición de la PIA vía método directo en vejiga no es factible. Este incluye paciente con ruptura de vejiga, hematoma intravesical, vejiga neurogénica, cirugía vesical reciente y anomalías urogenitales. La WSACS recomienda la medición de la PIA versus no medición cuando está presente cualquier factor de riesgo para HIA/SCA en pacientes críticamente enfermos o lesionados (Ver tabla 2). ${ }^{5,6}$

Tabla 2. Factores de riesgo para HIA/SCA

\begin{tabular}{|l|}
\hline \multicolumn{1}{|c|}{ FACTOR DE RIESGO } \\
\hline Disminución de la compliance de la pared ab- \\
dominal \\
Cirugía abdominal \\
Trauma y quemadura mayor \\
Posición prona \\
\hline Aumento del contenido intraluminal \\
Gastroparesis/distensión gástrica, íleo \\
Pseudoobstrucción colónica \\
Vólvulo \\
\hline Aumento del contenido intraabdominal \\
Pancreatitis aguda \\
Abdomen distendido \\
Hemoperitoneo/neumoperitoneo o colecciones \\
de líquido intraperitoneal \\
Infección intraabdominal/absceso \\
Tumores intraabdominales o retroperitoneales \\
\hline
\end{tabular}

\begin{tabular}{l}
\hline Laparoscopía con insuflación excesiva \\
Disfunción hepática/cirrosis con ascitis \\
Diálisis peritoneal \\
\hline Fuga capilar/resucitación con líquidos \\
Acidosis \\
Laparotomía de control de daño \\
Hipotermia \\
Score APACHE II o SOFA aumentados \\
Resucitación masiva con líquidos o balance de \\
líquido positivo \\
\hline Otras/misceláneos \\
Edad, bacteremia, coagulopatía, aumento del án- \\
gulo de la cabecera de la cama, reparación de \\
hernia incisional masiva, ventilación mecánica, \\
obesidad, PEEP > 10, peritonitis, neumonía, sep- \\
sis, shock o hipotensión. \\
Fuente: Evidence-Based Reviews in Surgery Vol. 218, No. 6, \\
June 2014
\end{tabular}

\section{Técnicas de cierre abdominal tempo-} ral

La necesidad del cierre abdominal temporal (CAT) es una consecuencia lógica de la descompresión abdominal o tratamiento profiláctico de abdomen abierto. Debido a que la utilización del manejo con abdomen abierto para prevenir o tratar el SCA está aumentando, el CAT se requerirá con mayor frecuencia, y todos los encargados del cuidado del paciente deben estar atentos a las indicaciones, complicaciones y dificultades en el manejo del abdomen abierto con técnicas de CAT.

Con mayor importancia, las diferentes especialidades involucradas en el cuidado del paciente deben tener una meta en común: cierre abdominal temprano.

Indicaciones de abdomen abierto y cierre abdominal temporal

Históricamente, el manejo con abdomen abierto ha sido utilizado en pacientes con sepsis abdominal que requieren relaparotomía planeada. Hoy en día, el OA se utiliza con mayor frecuencia para prevenir SCA posterior a una cirugía abdominal de emergencia por trauma, ruptura de aneurisma abdominal o enfermedad de víscera abdominal. La otra indicación importante es en el tratamiento de HIA o SCA. En la mayoría de casos, es una laparotomía en línea media que se deja abierta y 
requiere CAT. Rara vez el CAT es también requerido para defectos de la pared abdominal después de trauma o infecciones necrotizantes. La incisión en línea media que se deja abierta expone la cavidad peritoneal con el hígado, estómago, omento e intestino delgado en contacto directo con la herida. Inicialmente, la meta ideal de CAT era prevenir lesión a estos órganos y la contaminación de la cavidad abdominal estéril. A medida que aumenta la experiencia, el control de la pérdida de líquido, la prevención de la pérdida del dominio abdominal y evitar daño a la fascia que pueda afectar subsecuentemente el cierre abdominal, fueron también encontrados como metas importantes. Con la llegada de la terapia de presión negativa, la eliminación activa de líquido de la cavidad abdominal puede ser la próxima meta terapéutica.

\section{Problemas con el cierre abdominal temporal}

El manejo del abdomen abierto en la UCI puede ser complicado. La pérdida de líquido y calor pueden ocurrir, pero son manejables, y también la pérdida de proteína es un problema importante. Complicaciones como sangrado y fístulas son frecuentes; la incidencia de fístula se relaciona con la duración del tratamiento con abdomen abierto. La elección de la técnica de CAT puede afectar el tipo de complicación y el rango.

\section{Técnicas de cierre abdominal temporal}

La lista de características deseadas de la técnica ideal de CAT es larga y ninguno de los métodos actuales posee todas. La presión negativa es la más reciente mejoría en las técnicas de CAT; la eliminación activa de líquido del abdomen parece ser el próximo paso en el manejo de abdomen abierto.

La técnica de CAT ideal, debe tener las siguientes características: disponibilidad universal, facilidad y rapidez de aplicación, porosidad, capacidad de control de pérdida de líquido, prevención de SCA, que deje intacta la fascia y la piel, que no sea reactivo al intestino y otros órganos, fácil de remover y reemplazar, que mantenga la cavidad abdominal estéril y de bajo costo. Se categorizan las diferentes técnicas de CAT en tres generaciones, reflejando la evolución histórica, (ver tabla 3).

Tabla 3. Clasificación de las diferentes técnicas de CAT

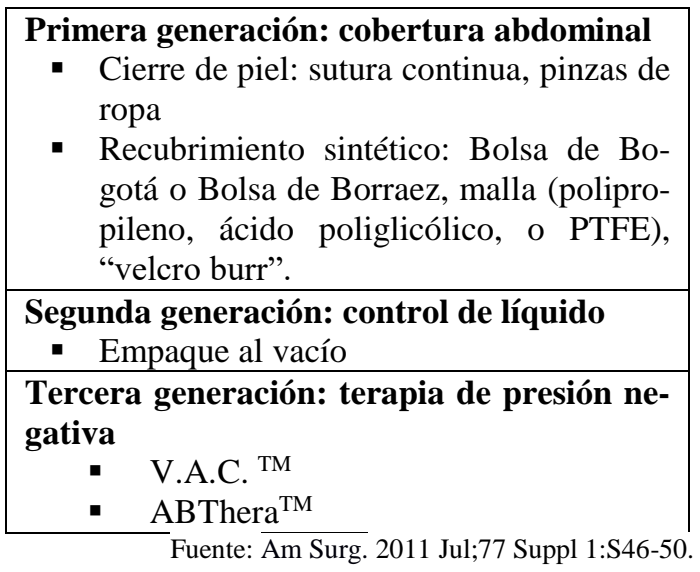

\section{Cierre abdominal temporal de pri- mera generación}

Cierre de piel

Es la forma más básica de CAT. Puede hacerse con suturas o con pinzas de ropa, (ver figura 3). Es barato, rápido y disponible universalmente pero daña la piel y puede ser insuficiente para prevenir SCA recurrente. No previene tampoco retracción de la fascia, y las pinzas pueden interferir con investigaciones radiológicas.

Figura 3. Cierre abdominal temporal con pinzas de ropa

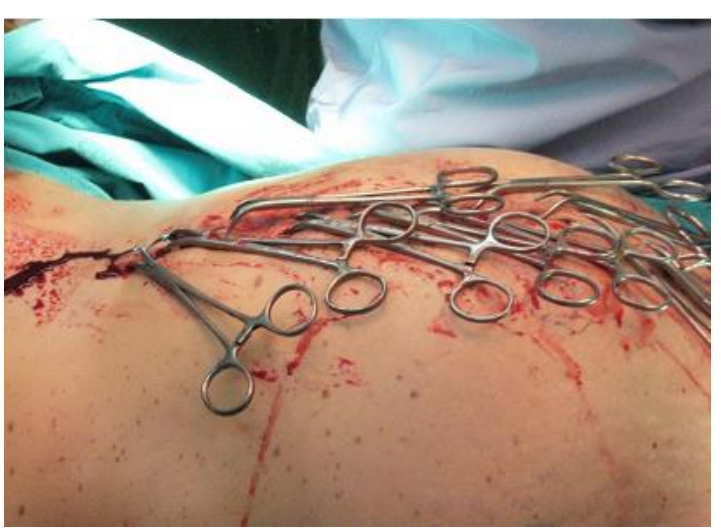

Fuente: Songkla Med J Vol. 32 No. 2 Mar-April 2014 


\section{Recubrimiento sintético}

Se introdujo primero por cirujanos colombianos, quienes utilizaron una simple hoja cortada de una bolsa de solución salina de 3L (Bolsa de Bogotá o Bolsa de Borraez) que se sutura a la fascia o la piel. Se pueden utilizar hojas de silicón también. Varias mallas caen dentro de esta categoría también, pueden ser absorbibles o no absorbibles: se han utilizada polipropileno (Marlex, Prolene, Surgipro), ácido poliglicólico (Dexon), poliglactin (Vicryl), y PTFE (Gore-Tex). No se ha reportado experiencias a gran escala con el uso de mallas biológicas. Otras próstesis se han desarrollado específicamente con este propósito, por ejemplo, el parche de Wittman ${ }^{\mathrm{TM}}$ que consiste en dos hojas de velcro, (ver figura 4).

\section{Figura 4. Cierre abdominal temporal con ma- Ila de poliglactin}

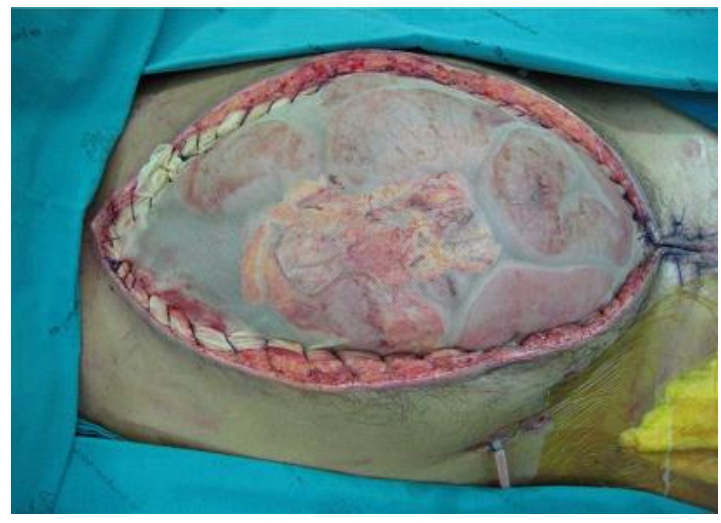

Fuente: Songkla Med J Vol. 32 No. 2 Mar-Apr 2014

\section{Cierre abdominal temporal de se-} gunda generación

Esta técnica se introduce para sobrellevar el problema de pérdida de líquido y la función de barrera incompleta observada en las técnicas de CAT de primera generación. Se colocaban drenos en la gasa que cubría los intestinos y se cubría la herida con envoltura adhesiva. Los drenos se conectaban a colectores de tubo de tórax, y aunque la presión resultante era baja $\left(20-25 \mathrm{cmH}_{2} \mathrm{O}\right)$, permitían un control fácil del acúmulo de líquido y prevenían fuga no controlada. Se utilizaron diferentes nombres para ese sistema, descritos por Brock et al. por primera vez; acuñado también como empaque de Barker, VacPack o técnica de sándwich de empaque al vacío.

\section{Cierre abdominal temporal de tercera generación}

La terapia de presión negativa (TPN) ha sido utilizada por algún tiempo en el manejo de varios tipos de heridas. El uso de este abordaje se introdujo apósito abdominal con VAC, que consiste en una hoja de plástico perforada que contiene una espuma, encima de la cual se inserta una esponja que cubre el defecto abdominal. Esto se cubre con un sello hermético y se conecta a una bomba.

Aparte de cubrir el abdomen, permite la evacuación del exceso de líquido abdominal y mantiene una tensión constante en la fascia. La experiencia clínica confirma que la TPN en este escenario es segura y a pesar de las preocupaciones por el desarrollo de fístulas intestinales, la incidencia reportada en revisiones recientes es la menor comparada a todas las técnicas de CAT.

La última versión de este apósito, el ABThera ${ }^{\mathrm{TM}}$, tiene "brazos" que hacen más fácil su inserción y mejora también la aplicación de la presión negativa a través de la superficie del abdomen. La combinación de TPN combinada con un sistema de CAT para aproximar progresivamente la fascia ha ido en aumento, (ver figura 5).

\section{Figura 5. Apósito abdominal con VAC}

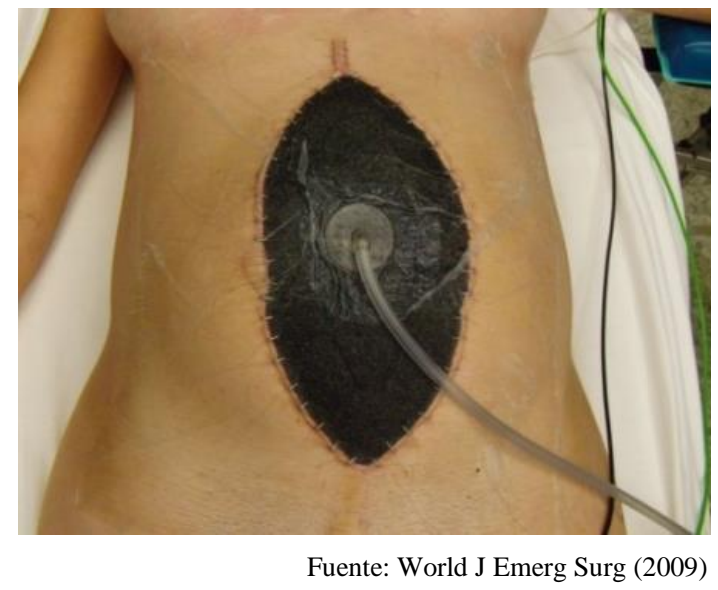




\section{Técnicas de aproximación de fascia}

Los métodos disponibles de CAT deben integrarse en una estrategia quirúrgica que facilite el cierre temprano. Diferentes modalidades que aumentan el rango de cierre primario están disponibles y están dirigidas primariamente a la prevención de la pérdida de dominio y el cierre progresivo, y proactivo de la herida. Preferiblemente, la tensión en los bordes de la fascia debe incrementarse durante el monitoreo intraoperatorio de la PIA.

\section{Cierre abdominal seriado y retención dinámica}

Estás técnicas, ambas utilizan una hoja protectora sobre las vísceras y suturas que se insertan a través de la piel, grasa y fascia. Estas suturas se protegen utilizando un tubo de tórax o sonda Foley que protege las vísceras de la erosión. Las suturas no se anudan pero se sujetan. Esto permite la aproximación progresiva de la fascia. Otros han utilizado mallas que pueden también acortarse progresivamente para aproximar la fascia. El parche de Wittman $^{\mathrm{TM}}$ se basa en el mismo principio y puede adaptarse fácilmente a las necesidades del paciente.

Cierre de herida asistido por vacío mediado con malla

Esta técnica utiliza una malla no reabsorbible en combinación con TPN. La malla se sutura a la fascia y yace entre una hoja protectora y la esponja en la herida de laparotomía. ${ }^{7}$

\section{Terapia de presión negativa (TPN)}

La TPN involucra la aplicación de algún grado de succión a una herida abdominal abierta. A través del uso de una cubierta y presión negativa constante en la herida, esta técnica previene la adherencia visceral a la pared abdominal anterolateral mientras mantiene la tracción medial de la fascia, lo que puede aumentar las tasas de cierre de fascia entre los que tienen un abdomen abierto. Puede también remover líquido y citoquinas proinflamatorias del peritoneo, lo que puede reducir el volumen del tercer espacio abdominal, la respuesta inflamatoria sistémica y disfunción orgánica resul- tante. Sin embargo, otros han reportado preocupaciones por asociaciones entre la TPN y SCA recurrente o fístula intestinal o enteroatmosférica, especialmente entre aquellos con líquido intraabdominal limitado disponible para remover. A pesar de la variedad de métodos de CAT, el consenso del 2013 de la WSACS realiza una recomendación fuerte de utilizar TPN en pacientes críticos con abdomen abierto, basado en evidencia de bajo grado. ${ }^{6,8}$

Cirocchi et al en 2016 publican un meta-análisis y revisión sistemática con investigación de literatura de MEDLINE, PubMed, y las bases de datos de la librería de Cochrane, de acuerdo con la declaración PRISMA y el Manual Cochrane para las Revisiones Sistemáticas. El objetivo del estudio es comparar publicaciones de técnicas con presión negativa y sin presión negativa, con el fin de evaluar si alguna técnica se asocia con mejores resultados que otras, en términos de rango de cierre primario de fascia, fístula enteroatmosférica, absceso abdominal, hemorragia, duración de la estancia en UCI y morbilidad y mortalidad general posoperatoria. Los autores reportan:

\section{Cierre primario de fascia $(C P F)$}

Chen et al. reportan rangos de complicación y mortalidad significativamente reducidos en los casos con $\mathrm{CPF}$, independientemente de la técnica de CAT, lo que fue confirmado por otros. En general, el rango de CPF en TPN fue menor en todos los estudios, excepto en el estudio de $\mathrm{Mu}-$ tafchiyski et al, dónde los autores reportaron $73 \%$ de CPF pero en combinación con tracción mediada con malla.

Los dos estudios randomizados controlados fueron pobres en calidad y el análisis de sensibilidad no reveló diferencia estadísticamente significativa. El estudio prospectivo observacional de Carlson et al. es el único que reporta un rango significativamente menor de CPF en TPN con $16 \%$ con análisis emparejado. Basados en experiencia propia y en la literatura publicada, los autores concluyen que la TPN por sí misma no es suficiente para lograr un rango mayor de CPF y su combinación con una de las técnicas de cierre dinámico de fascia es altamente recomendado. Peterson, 
Acosta y Börck de Suiza realizaron la primera descripción de cierre dinámico de fascia con tracción mediada con malla combinada con VAC. Ellos utilizaron malla de polipropileno suturada a la fascia con tensión gradual posterior a cada revisión. Los autores reportaron CPF en los siete pacientes después de una media de 9 procedimientos de tensión de malla con una media de 32 días sin recurrencia de SCA, y con cero mortalidad. El meta-análisis de Atema et al. parece apoyar la efectividad de esta técnica.

\section{Rango de complicaciones generales al día 30 del posoperatorio}

A pesar de sus beneficios, el abdomen abierto puede estar asociado con serias complicaciones. Sin embargo, el rango de complicaciones generales al día 30 del posoperatorio no es frecuentemente reportado. Solamente un estudio reportó la morbilidad general a 30 días del posoperatorio. Rencuzogullari et al reportaron un rango menor de morbilidad en el grupo de VAC, pero no fue estadísticamente significativo. Dadas las limitaciones de su estudio, Carslon et al no encontró diferencias significativas entre TPN y no TPN.

\section{Fístula enteroatmosférica posoperatoria}

La fistula enteroatmosférica (EAF) continúa siendo un reto serio para los pacientes, cirujanos y equipo de enfermería. Son una complicación típica y devastadora del abdomen abierto. Fisher et al encuentran un rango significativamente mayor de fístula enteroatmosférica en abdomen abierto comparado con pacientes que se sometían a cierre definitivo $(6.8 \%$ vs $0.6 \%)$.

Miller et al reportan un rango de $75 \%$ de fístula en los casos con cierre abdominal retardado. La EAF puede llevar a una estancia en UCI y hospitalaria en general significativamente prolongada, aumento de los costos hospitalarios, rangos de CPF menores y a un aumento directo en la mortalidad. La hipótesis inicial de la posible causa de la presión negativa no fue apoyada por el trabajo de varios autores.

El análisis conjunto reveló un menor rango de EAF con TPN, mientras que los análisis de sensibilidad de los estudios randomizados controlados encontró más EAF en esta situación. Sin embargo, en ambos casos las diferencias no fueron estadísticamente significativas. A pesar del modelo al azar utilizado el análisis reveló efectos en direcciones opuestas y similares al análisis de CPF.

Esta heterogeneidad sustancial dificulta significativamente la interpretación de este hallazgo. El uso de una barrera no adherente entre el intestino y la pared abdominal parece contribuir a rangos menores de EAF a través de prevención de adherencias, pero sin diferencia significativa entre las diferentes técnicas de TPN. Medidas importantes de prevención incluyen evitar re-exploraciones frecuentes y realizar todos los esfuerzos por CPF temprano, técnica quirúrgica delicada y uno de los principales principios del triage de combate-"el mismo concepto de equipo".

En caso de formación de EAF, artículos recientes sugieren el aislamiento y la exteriorización cuidadosa de la EAF para obtener un drenaje efectivo de los fluidos intestinales. Obtener un control y una diversión adecuados del drenaje de la fistula se considera esencial para una granulación limpia del intestino expuesto y la epitelización del abdomen.

\section{Absceso posoperatorio}

Unas de las ventajas atribuidas a la TPN es la mejor evacuación de los líquidos intraabdominales, lo que puede contribuir a un menor rango de abscesos intraabdominales particularmente en el escenario de un abdomen séptico. El análisis de Cirocchi incluyó solo dos estudios con heterogeneidad aceptable y no encontró un rango significativamente menor en el grupo de TPN. Este resultado puede ser también erróneo pues los rangos de absceso dependen también de la frecuencia y la calidad de las re-exploraciones. En el grupo TPN no hubo diferencia entre ABThera y el empaque al vacío de Barker.

\section{Sangrado posoperatorio}

El sangrado no es una complicación reportada con frecuencia en abdomen abierto. Teóricamente se ha planteado como hipótesis que los pacientes con TPN pueden estar predispuestos a un rango mayor 
de sangrado que aquellos sin TPN. Desafortunadamente, los reportes de complicaciones en abdomen abierto usualmente no comentan sobre esta complicación. Los datos obtenidos por Cirocchi et al demuestra un rango de sangrado levemente menor pero estadísticamente no significativo en TPN, aunque los dos estudios consultados presentaban resultados contradictorios.

Carslon et al demostraron un rango insignificantemente mayor de intervención para controlar sangrado en el grupo de no TPN, mientras que más recientemente Szmyt et al reportaron en una pequeña serie de casos, un solo evento de sangrado en TPN comparado con ningún sangrado en pacientes sin TPN. Por lo que la información disponible es insuficiente para establecer una conclusión significativa.

\section{Mortalidad a los 30 días del posoperatorio}

El rango de mortalidad general en el trabajo de Cirocchi et al fue significativamente menor en el grupo de TPN en el escenario de heterogeneidad significativa. Este resultado debe ser interpretado con cautela debido a la naturaleza compleja de la medida de este resultado, influenciada por otros factores como el tipo de CAT, edad del paciente, presencia de comorbilidades, la severidad de la patología subyacente y el rango de CPF.

Por otro lado, cuando se compararon técnicas hubo diferencias significativas entre las técnicas de TPN. El subgrupo de análisis encontró una menor mortalidad después de la aplicación del sistema $\mathrm{ABThera}^{\mathrm{TM}}$ comparado con el empaque al vacío de Barker. El estudio controlado de Cheatham et al. encontró una posibilidad de supervivencia significativamente mayor con ABThera.

\section{Duración de la estancia hospitalaria en UCI al posoperatorio}

Cirocchi et al encuentran una estancia significativamente menor en UCI con TPN con una diferencia media de 4.5 días. Un paso importante hacia esta dirección es el uso de cierre dinámico de fascia lo cual ha probado reducir la estancia en general. ${ }^{9}$

\section{Sistema ABThera ${ }^{\mathrm{TM}}$ versus Sistema VAC abdominal}

Los dos sistemas de apósitos con presión negativa más utilizados son el sistema VAC abdominal y el sistema ABThera de KCI. El sistema de apósito abdominal consiste en una esponja envuelta en plástico diseñada para estar en contacto directo con las vísceras.

El sistema ABThera utiliza la misma técnica con refinamientos mejorados. Consiste en una capa grande protectora de vísceras que incluye una esponja central cubierta con poliuretano con seis brazos de esponja de poliuretano que se extienden desde el centro para envolver las vísceras al extenderse profundo en las goteras paracólicas y drenar líquido de estos espacios, la pelvis y entre las asas intestinales. ${ }^{10}$

Figura 6. Sistema ABTheraTM

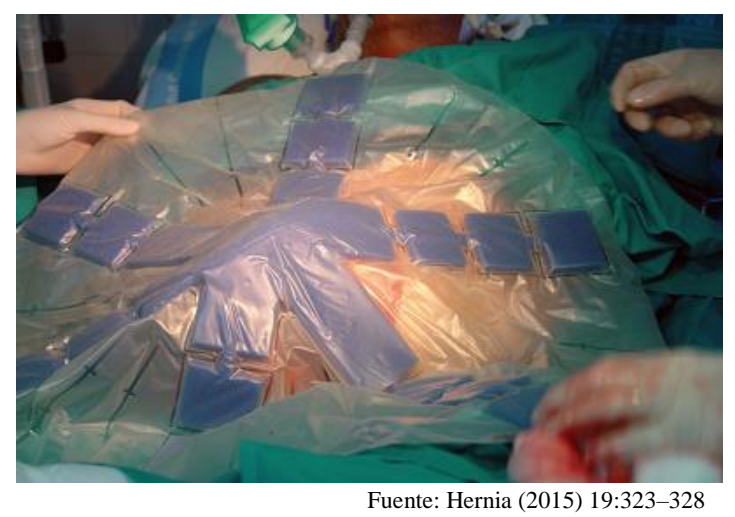

Cheatham et al realizan un estudio abierto prospectivo, observacional para evaluar dos técnicas de CAT en pacientes quirúrgicos y de trauma que requerían manejo con abdomen abierto: Técnica de Barker de empaque al vacío (BVPT) y el sistema ABThera ${ }^{\mathrm{TM}}$.

Los criterios de valoración fueron rango y días de cierre primario de fascia (CPF) y mortalidad de cualquier causa a 30 días. Se incluyeron 283 pacientes de 20 centros de trauma de Estados Unidos entre el 2009 y el 2011 que cumplían con los criterios de inclusión. El cierre primario de fascia a los 30 días ocurrió en 139 (78\%) pacientes de 178 tratados con ABThera versus $69(68 \%)$ pacientes 
de 102 tratados con BVPT. El rango de complicaciones se observa en la siguiente tabla:

Tabla 4. Complicaciones del manejo con el sistema $A B T h e r a{ }^{T M}$ versus BVPT

\begin{tabular}{|c|c|c|c|}
\hline Complicación & ABThera & BVPT & $p$ \\
\hline $\begin{array}{l}\text { Infección/abs- } \\
\text { ceso abdominal }\end{array}$ & $23 \%(40)$ & $\begin{array}{l}26 \% \\
(26)\end{array}$ & 0.56 \\
\hline SCA & $8 \%(14)$ & $8 \%(8)$ & 1.00 \\
\hline $\begin{array}{l}\text { Dehiscencia de } \\
\text { herida abdomi- } \\
\text { nal }\end{array}$ & $2 \%(3)$ & $1 \%(1)$ & 1.00 \\
\hline $\begin{array}{l}\text { Fuga anastomó- } \\
\text { tica }\end{array}$ & $4 \%(7)$ & $2 \%(2)$ & 0.49 \\
\hline $\begin{array}{l}\text { Erosión del sitio } \\
\text { de aplicación }\end{array}$ & $0 \%$ & $1 \%(1)$ & 0.36 \\
\hline Coagulopatía & $5 \%(9)$ & $5 \%(5)$ & 1.00 \\
\hline $\begin{array}{l}\text { Trombosis ve- } \\
\text { nosa profunda }\end{array}$ & $3 \%(5)$ & $4 \%(4)$ & 0.73 \\
\hline Necrosis fascial & $2 \%(4)$ & $5 \%(5)$ & 0.29 \\
\hline $\begin{array}{l}\text { Isquemia gas- } \\
\text { trointestinal }\end{array}$ & $7 \%(13)$ & $3 \%(3)$ & 0.18 \\
\hline $\begin{array}{l}\text { Fistula intesti- } \\
\text { nal }\end{array}$ & $4 \%(7)$ & $4 \%(4)$ & 1.00 \\
\hline $\begin{array}{l}\text { Obstrucción } \\
\text { intestinal }\end{array}$ & $3 \%(5)$ & $0 \%$ & 0.16 \\
\hline $\begin{array}{l}\text { Falla multior- } \\
\text { gánica }\end{array}$ & $8 \%(15)$ & $\begin{array}{l}16 \% \\
(16)\end{array}$ & 0.08 \\
\hline $\begin{array}{l}\text { Embolismo pul- } \\
\text { monar }\end{array}$ & $1 \%(1)$ & $0 \%$ & 1.00 \\
\hline
\end{tabular}

La mortalidad a los 30 días por cualquier causa ocurrió en $21(12 \%)$ de 178 pacientes del grupo del sistema ABThera $^{\mathrm{TM}}$ y en 20 (20\%) de 102 pacientes manejados con BVPT. Este es el primer estudio que demuestra una ventaja de supervivencia asociada con una técnica de CAT en particular. Olona et al. realizan un estudio retrospectivo comparativo de pacientes tratados con abdomen abierto y TPN entre Enero de 2006 y Enero de 2013, en la unidad quirúrgica digestiva y general del Hospital Universitario Joan XXIII de Tarragona.

El objetivo fue comparar el tiempo de cierre de pared abdominal con el uso del sistema ABThera versus apósito abdominal. Se encontraron un total de 73 pacientes tratados con TPN. El tiempo promedio de cierre abdominal fue de 26 días en el grupo de apósito abdominal (AD) y 17 días en el grupo ABThera, la media de cambio de apósitos fue de 8 en el grupo AD y 5 en el grupo ABThera, con diferencias estadísticamente sigfinicativas.

El cierre primario de fascia se realizó en 5 (11\%) de pacientes tratados con $\mathrm{AD}$ y $11(41 \%)$ tratados con ABThera, con diferencias estadísticamente sigfinicativas. Fístula, clasificada como Clavien grado III se observó en 8 pacientes (20\%) en el grupo $\mathrm{AD}$ y solo un paciente (4\%) en el grupo ABThera, con diferencias no sigfinicativas estadísticamente. Durante el seguimiento 15 pacientes (33\%) en el grupo AD y 4 pacientes (15\%) en el grupo ABThera murieron debido al empeoramiento de la condición subyacente. ${ }^{11,12}$

\section{CONCLUSIONES}

La utilización de la técnica de abdomen abierto en el manejo de situaciones quirúrgicas críticas representa un reto para el cirujano y para todo el equipo de atención del paciente. A lo largo de las historia se han desarrollado múltiples dispositivos para acelerar el proceso de cierre de la pared abdominal con el objetivo de reducir la estancia hospitalaria y las severas complicaciones que puede presentar el paciente con abdomen abierto.

A pesar de la amplia gama de opciones la terapia de presión negativa ha recibido la mayor atención en los últimos años, siendo recomendada en el 2013 por la Sociedad Mundial del Síndrome Compartimental para manejar al paciente crítico, a pesar de tener nivel de evidencia bajo.

Diferentes estudios han concluido que no existe evidencia suficiente que establezca beneficios significativos con la terapia de presión negativa en cuanto a cierre primario de fascia, mortalidad, complicaciones, hemorragia, formación de absceso abdominal y estancia hospitalaria.

Finalmente, los dos sistemas de apósitos con presión negativa más utilizados son el sistema VAC abdominal y el sistema ABTheraTM, que en estudios comparativos de estas dos técnicas se atribuye una ventaja de supervivencia con el uso del sistema ABTheraTM. 


\section{BIBLIOGRAFÍA}

1. Navsaria P, Nicol A, Hudson D, Cockwill $\mathrm{J}$, Smith J. Negative pressure wound therapy management of the "open abdomen" following trauma: a prospective study and systematic review. World J Emerg Surg. 2013; $8(1): 4$

2. Atema JJ Gans SL Boermeester MA. Systematic review and meta-analysis of the open abdomen and temporary abdominal closure techniques in non-trauma patients. World J Surg. 2015; 39(4):912-25

3. Kreis BE de Mol van Otterloo AJ Kreis RW. Open abdomen management: a review of its history and a proposed management algorithm. Med Sci Monit. 2013; 19:524-33

4. Coccolini FBiffl W Catena F et al. The open abdomen, indications, management and definitive closure. World J Emerg Surg. 2015; 10: 32 .

5. Hunt L A Frost S Hillman K Newton P Davidson P. Management of intra-abdominal hypertension and abdominal compartment syndrome: a review. J Trauma Manag Outcomes. 2014; 8: 2.

6. Andrew W. Kirkpatrick A Roberts D De Waele J et al. Intra-abdominal hypertension and the abdominal compartment syndrome: updated consensus definitions and clinical practice guidelines from the World Society of the Abdominal Compartment Syndrome. Intensive Care Med. 2013 Jul; 39(7): 1190_ 1206.

7. De Waele JJ Leppäniemi AK. Temporary abdominal closure techniques. Am Surg. 2011; 77(1): 46-50.

8. Hecker A Hecker B Hecker M Riedel JG Weigand MA Padberg W. Acute abdominal compartment syndrome: current diagnostic and therapeutic options. Langenbecks Arch Surg. 2016 Feb; 401(1):15-24
9. Cirocchi R Birindelli A Biffl WL et al. What is the effectiveness of the negative pressure wound therapy (NPWT) in patients treated with open abdomen technique? A systematic review and meta-analysis. J Trauma Acute Care Surg. 2016.

10. Huang Q Li J, Lau WY. Techniques for Abdominal Wall Closure after Damage Control Laparotomy: From Temporary Abdominal Closure to Early/Delayed Fascial Closure-A Review. Gastroenterol Res Pract. 2016; 2016:2073260

11. Cheatham M Demetriades D Fabian $\mathrm{T}$ et al. Prospective Study Examining Clinical Outcomes Associated with a Negative Pressure Wound Therapy System and Barker's Vacuum Packing Technique. World J Surg. 2013; 37(9): 2018-2030.

12. Olona C Caro A Duque E et al. Comparative study of open abdomen treatment: ABThera ${ }^{\mathrm{TM}}$ vs. abdominal dressing ${ }^{\mathrm{TM}}$. Hernia. 2015; 19(2): 323-328

\section{CONFLICTO DE INTERÉS Y/O AGRADE- CIMIENTOS}

Los autores declaran que no existió ningún conflicto de interés en el presente reporte. 
Figura 1. Diagrama de manejo de abdomen agudo

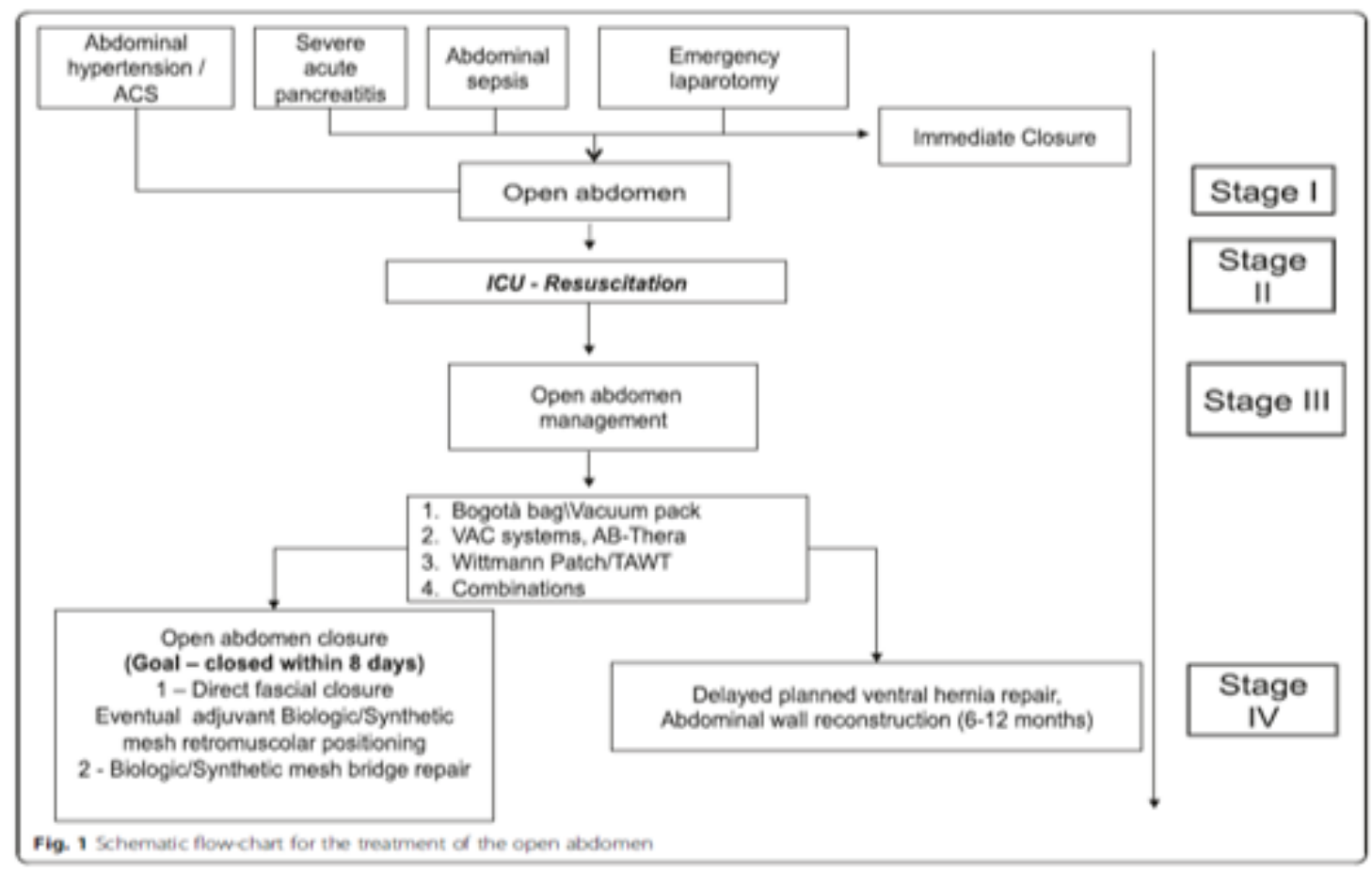

Fuente: Coccolini et al. World Joumal of Emergency Surgery (2015) 10:32 
Tabla 1. Efectos de la HIA en varios ôrganos sistémicos y manifestaciones clínicas del SCA

\begin{tabular}{|l|l|l|}
\hline Sistema & Efecto & Manifestación \\
\hline Renal & $\begin{array}{l}\text { Compresión de vena renal, compresión } \\
\text { arteriolar cortical. }\end{array}$ & Oliguria, creatinina en aumento \\
\hline Pulmonar & $\begin{array}{l}\text { Presión en aumento en el diafragma, } \\
\text { disminución de la compliance y de la capacidad } \\
\text { residual, aumento en la resistencia de la vía } \\
\text { aérea. }\end{array}$ & $\begin{array}{l}\text { Hipoxia, hipercarbia, presión } \\
\text { elevada en la vía aérea, disminución } \\
\text { del volumen tidal. }\end{array}$ \\
\hline Cardiovascular & $\begin{array}{l}\text { Disminución del retorno venoso, aumento de la } \\
\text { poscarga }\end{array}$ & Disminución del gasto cardiaco \\
\hline Cerebral & $\begin{array}{l}\text { Aumento de la presión intratorácia con } \\
\text { disminución de la salida del flujo cerebral }\end{array}$ & Elevación de la presión intracraneal \\
\hline Esplácnice & $\begin{array}{l}\text { Disminución de la perfusión hepática e } \\
\text { intestinal }\end{array}$ & $\begin{array}{l}\text { Acidosis metabólica, isquemia } \\
\text { intestinal }\end{array}$ \\
\hline
\end{tabular}

Fuente: tomado y modificado de Cescelini et al. World Journal of Emergency Surgery (2015) 10:32 